\title{
Modeling the Substrate Effects on Nanoindentation Mechanical Property Measurement
}

\author{
Mario Gonzalez, Kris Vanstreels and Adam M. Urbanowicz \\ IMEC, Kapeldreef 75, \\ B-3001, Leuven, Belgium \\ e-mail: mario.gonzalez@imec.be
}

\begin{abstract}
Nanoindentation technique is commonly used to characterize the mechanical properties of thin films. However, the validity of the measurements is greatly affected by the indentation depth and the substrate properties. The purpose of this study is to understand the influence of the substrate properties, the thin film thickness and the mechanical properties of the thin film itself on force-displacement curves obtained by nanoindentation.

The experimentally obtained force versus indentation depth curves of single and bi-layer thin film systems on silicon wafers were simulated using Finite Element Modeling (FEM). The materials properties of the thin film layers were extracted by fitting the load-displacement curves obtained by FEM and experiments.

The experimental part of this study includes nanoindentation tests performed on low-k films deposited on a silicon substrate. These films were treated during different periods of time $(0 \mathrm{~s}, 20 \mathrm{~s}, 35 \mathrm{~s}$, $70 \mathrm{~s}, 140 \mathrm{~s}, 350 \mathrm{~s}$ and $700 \mathrm{~s}$ ) with $\mathrm{He} / \mathrm{H} 2$ downstreamplasma (DSP) at a fixed temperature of $280^{\circ} \mathrm{C}$, thereby resulting in a double layered structure with the same total film thickness, but with different thickness and mechanical properties of the "modified" top layer. The results of this work provide considerable insight for the determination of the mechanical properties of layered systems.
\end{abstract}

\section{Introduction}

There is a clear trend in the microelectronic products toward higher functionality and increased performance. Additionally, there is an increase in complexity due to the need of miniaturization and higher reliability. To accomplish these challenges, new materials are being introduced to the industry with specific processing capabilities and mechanical properties.

Characterization of mechanical properties such as Young's modulus, yielding stress, fracture toughness, among others, must be done in order to anticipate and avoid failures during processing or service life. Due to the smaller scales, the characterization of the mechanical properties is more complicated than ever before. Different methods for characterizing the mechanical properties of materials have been frequently discussed in the literature [1-5].
Nanoindentation test is very popular in the industry to evaluate the mechanical properties of thin films, particularly elastic modulus, hardness, scratch resistance and time dependent properties among others [5-8]. Although such tests are relatively simple and fast to implement, there are many factors that make difficult to interpret the testing results.

One of the main issues affecting the measurement of properties of the thin film is the effect of the substrate [9-10]. Many investigators have proven that the tip geometry of the indenter, the penetration depth, pile-up and sink-in of materials or even, the instrument compliance affect the measurements. A common practice is to consider that the modulus of the thin film is not affected by the substrate for indentation depths smaller than $1 / 10$ of the film thickness. However, this rule of thumb is not valid for all the film/substrate systems because it does not take into account the relative stiffness between the thin film and the substrate.

Numerous analytical and Finite Element Modeling (FEM) techniques have been used to analyze the loaddisplacement data obtained from nanoindentation experiment, generally as a tool to understand the indentation process itself. This paper presents an overview of simulation results and nanoindentation tests, demonstrating how the properties of the thin film and the substrate can impact the indentation results. The effect of film thickness, indentation depth and substrate properties were studied. As case study, a low-k film deposited on a silicon substrate has been analyzed. These films followed a special plasma treatment resulting in a modification of the top layer, and therefore, forming a double layered structure.

\section{Nanoindentation tests}

All nanoindentation tests were carried out at room temperature using a nanoindenter XP system, from MTS System Corporation, equipped with a dynamic contact module and continuous stiffness measurement (CMS) option. The indentation loading and unloading scheme was similar for all tests, with a constant indentation strain rate of $0,05 \mathrm{~s}^{-1}$. At the maximum indentation depth, the load was kept constant for $10 \mathrm{sec}$. to identify the presence of creep. For the samples studied here, no significant creep was observed. Continuous stiffness measurements were carried out during the loading and peak-load holding with a superimposed $1 \mathrm{~nm}$ amplitude sinusoidal oscillation at $75 \mathrm{~Hz}$. As the Berkovich diamond 
indenter tip is pressed into the sample, the CSM stiffness data along with the penetration (h) and applied load (P) are simultaneously recorded. From the experimentally obtained load-displacement curve, the elastic modulus (E) and hardness $(\mathrm{H})$ of the low-k dielectric films can be calculated based on their relationship to contact area and the measured contact stiffness (S) [5]. The calibration of the system (Berkovich indenter tip shape and column compliance) was done on a fused silica sample and the proprietary MTS software.

\section{Finite Element Modeling}

For this study, a single thin film and a bi-layer system on a substrate as shown in Figure 1 will be analyzed by FEM. The thickness of the thin film layers and the properties are variables, thus a parametric study is feasible with minimum changes in this model.
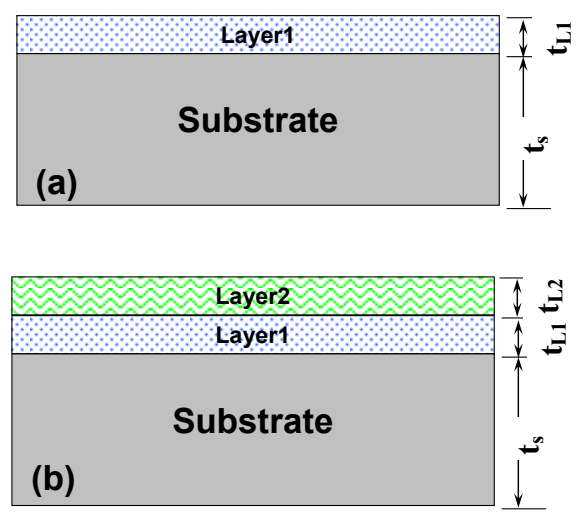

Figure 1. Schematic material system . (a) Thin film on substrate; (b) Bi-layer thin film on substrate.

It has been shown before [10] that a two dimensional FE analysis is sufficient to reproduce loadindentation depth curves with sufficient accuracy combined with high computational efficiency. The 2D-axisymmetric mesh consists of four-node axisymmetric elements as depicted in Figure 2. The contact region between the indenter tip and the thin film are finely meshed in order to insure the simulation accuracy at low indentation depths. Due to the large deformation occurring during the indentation process, the region below the indenter tip follows an adaptive mesh to avoid highly distorted elements during the indentation process. For this study, the commercial finite-element code MSC.MARC $\AA$ was used for the indentation simulations.

In this model a conical shape indenter is used with an angle of $70.3^{\circ}$ representing the same depth-to-area relation of the $3 \mathrm{D}$ Berkovich indenter. The radius of the conical indenter was considered to be $100 \mathrm{~nm}$. A rigid surface is used to model the conical indenter. The indenter is constrained to move in the thickness direction with a displacement control at a constant strain rate. The reaction force of the system and the displacement of the indenter are taken at each increment and it represents the material response to the indentation. The 3D FE simulations are performed for both loading and unloading processes in order to compare with the experimental results whenever it is possible.

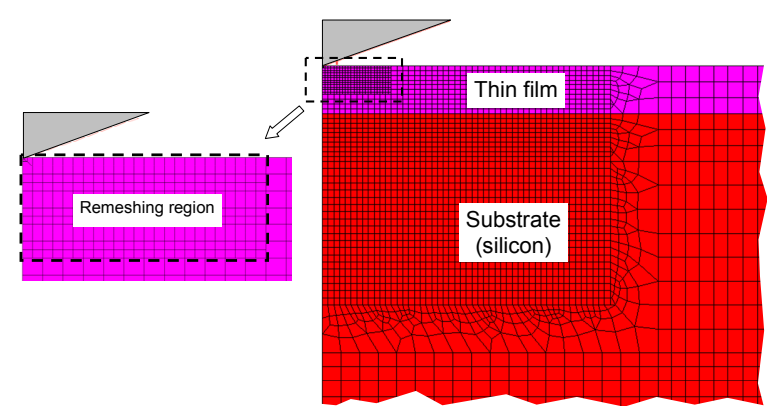

Figure 2. Two dimensional axi-symmetric model of a nanoindentation

The mechanical behavior of the silicon substrate is modeled as isotropic, linear-elastic. The thin film was modeled as isotropic, elastic-plastic solid. No hardening effect is included in the model because the first intention is not the modeling of metals and it is known that amorphous or non metallic materials have a negligible work hardening. However, the model is easily modified to include hardening. The interface between the thin film and the substrate is considered as perfectly bonded, therefore, no delamination is allowed.

\section{Parametric FE indentation simulations}

Parametric FE simulations were carried out in order to study the effect of material's properties on the forcedisplacement curves. In a first instance, two parameters were varied: the unitless Young's modulus ratio $\left(E_{\text {film }} / E_{\text {susbstrate }}\right)$ and the yield stress of the film $\left(\sigma_{y}\right)$. In all cases, the simulations consider a thin film of 500 $\mathrm{nm}$ deposited on a silicon substrate. Figure 3 shows the variation of the force displacement curve regarding the elastic modulus ratio. In this case, the Young's modulus of the thin film is varied from $84.5 \mathrm{GPa}$ to $507 \mathrm{GPa}\left(\mathrm{E}_{\mathrm{s}} / \mathrm{E}_{\mathrm{f}}\right.$ from 0.5 to 3$)$ and the substrate properties were kept constant. As it was expected, when the ratio $E_{f} / E_{s}$ increases, the required indentation force also increases. However, for the same penetration depth, this increase of the indentation force is not linearly dependent on the stiffness of the thin film. In the case of relatively soft films, small variations in the elastic modulus are reflected in large variations of the indentation force. This is because the "hard" substrate prevents the softer film from deforming downwards and thus increases the pile-up, overestimating, in consequence, the measured hardness of the material. Small variations in the load-displacement curves are observed when the thin film is equal or harder than the 
substrate. The effect of the substrate becomes less important when a thin film is stiffer than the substrate.

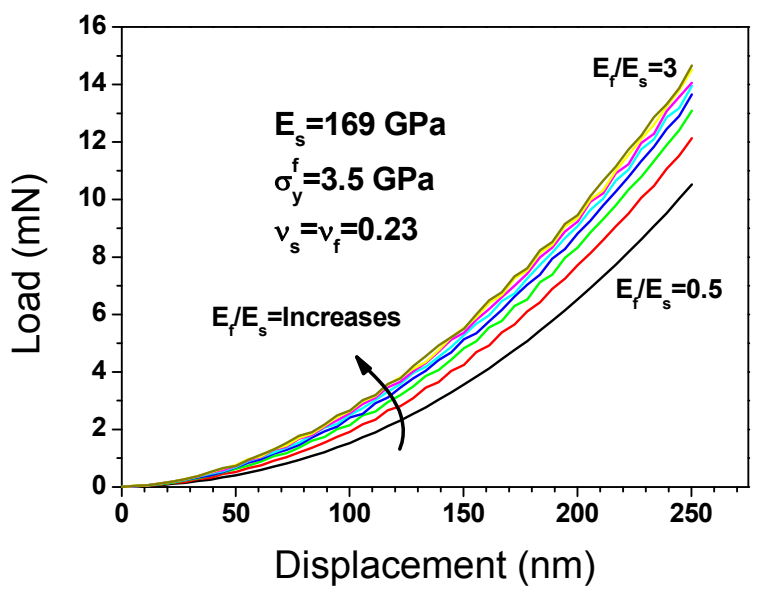

Figure 3. Influence of the elastic modulus ratio on the force-displacement response for the nanoindentation of a thin layer on a substrate

Figure 4 shows the von Mises stress distribution in the indentation region for the system soft-on-stiff when the indenter is at $25 \%$ of the film thickness. The region of the highest stress is beneath the indenter, however, it is visible an interaction of the stress field with the boundaries of the thin film, suggesting a small effect of the substrate at that indentation depth.

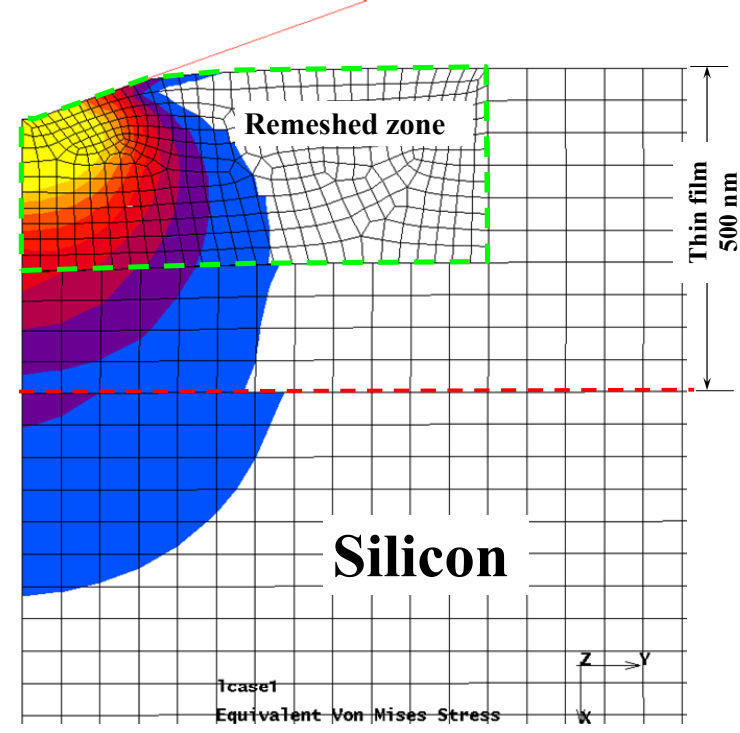

Figure 4. Von Mises Stress distribution of a soft thin film deposited on silicon. The maximum stress is located in the region below the indenter tip.

A similar approach was done for a bi-layer system, where two thin film layers of $500 \mathrm{~nm}$ where deposited on top of a silicon substrate. The Young's modulus of the silicon substrate and the first thin film layer were kept constant to 169 and $3 \mathrm{GPa}$ respectively. The elastic modulus of the top layer was varied from 1.5 to $12 \mathrm{GPa}$. Similar to the uni-layer system, the indentation load is shifted upwards when the elastic modulus ratio is increased. Opposed to the single layer case, for the range of properties and dimensions studied, the variation of the load-displacement curves are also observed for elastic modulus ratios of 4 or more as depicted in Figure 5.

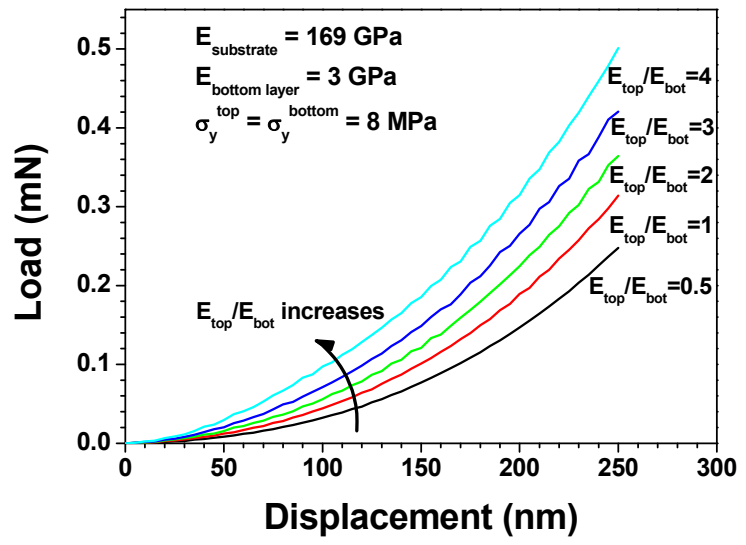

Figure 5. Influence of the elastic modulus ratio on the force-displacement response for the nanoindentation of a bi-layer system

The effect of the yield stress of the film is presented in Figure 6. Keeping constant the Young's modulus ratio $\left(E_{f} / E_{s}\right)$ and varying only the yield stress of the film, it is observed that an increase of this later will shift the load-displacement curve upward. This phenomenon can be explained by the larger elastic part at high yield points.

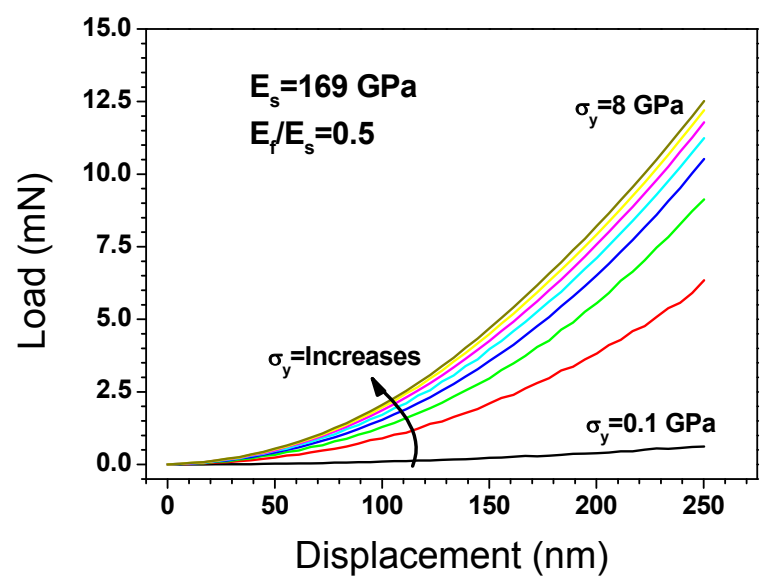

Figure 6. Influence of the yield stress on the Forcedisplacement response for a thin film on a substrate system

From the previous series of models we learned that the same load-displacement curve can be reproduced by different combinations of Young's modulus and yield stress. In other words, an experimental loading curve cannot be fitted with a single combination of Young's modulus and yield stress. In order to validate the previous statement, firstly, the loading $(P)$ versus displacement $(h)$ of a nanoindentation test done on 
fused silica was fitted with a quadratic expression $\left(P=A h^{2}\right)$, where $A$ is the loading curvature. Secondly, a design of experiment (DOE) was created, with the elastic modulus and yield stress of the substrate as variables. A quadratic fitting was also done on the force-displacement modeling results and the fitting coefficient, $A$, was compared. The best fitting curve was obtained when both fitting coefficients are equal.

Figure 7 depict a Response Surface Model (RSM) showing the relation between Young's modulus and yield stress of the fused silica sample. When the difference between the loading curvature, $A$, of the experimental curve and the FEM curve is zero means that the combination of elastic modulus and yield stress reproduce the same load-displacement curve as the one obtained experimentally. The combination of $\mathrm{E}$ and $\sigma_{\mathrm{y}}$ reproducing the same load-displacement curve is represented by the intersection between the RSM and the virtual XY plane depicted in Figure 7.

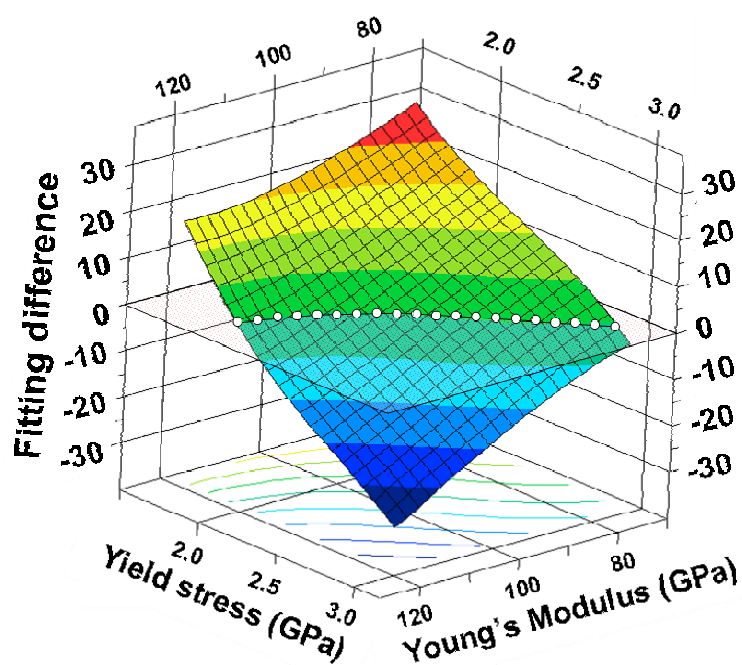

Figure 7. Response surface model

Although the loading curve is well reproduced with different combinations of elastic modulus and yield stresses, the unloading curve, in theory, is only sensitive to the elastic modulus. Therefore, in order to retrieve the best fitting parameters, both, the loading and unloading curves need to be fitted. In practice, it is not always possible to obtain a proper unloading curve, especially in brittle materials, because of the small cracks produced during the indentation. In other cases, a delamination at the interfaces of different materials can also affect the initial slope of the unloading curves.

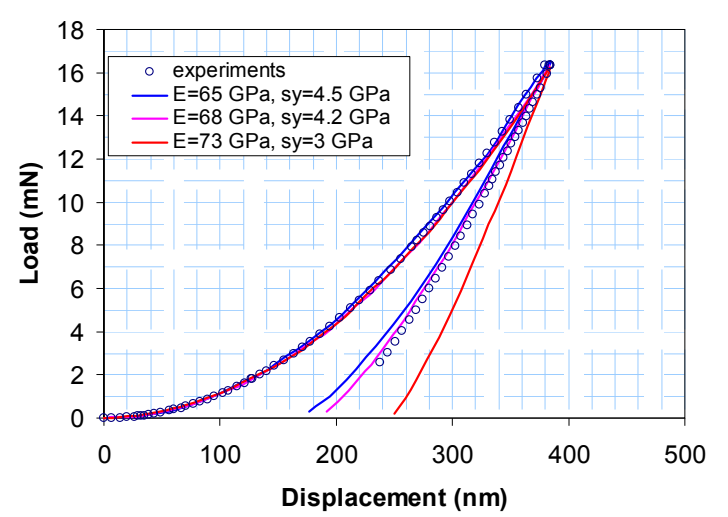

Figure 8. Experimental and FEM load-displacement curves for a fused silica calibration sample.

\section{Case study: Nanoindentation of a bi-layer low-k} film.

The mechanical properties of a porous low-k film, with $180 \mathrm{~nm}$ in thickness, were examined using nanoindentation with continuous stiffness measurement technique. The films were deposited on a silicon substrate and then were treated with a $\mathrm{He} / \mathrm{H}_{2}$ downstream-plasma (DSP) at a fixed temperature of $280^{\circ} \mathrm{C}$ and different exposure times. This special treatment results in an increase of the porosity in the top region of the film, and therefore, the mechanical behavior of the modified layer is changed and a double layer system is created [11]. The thickness of the modified top layer varied with the time, but the total film thickness remains constant as depicted in Figure 9. The porosity increase is related to the elimination of porogen residues formed during ultra-violet curing.

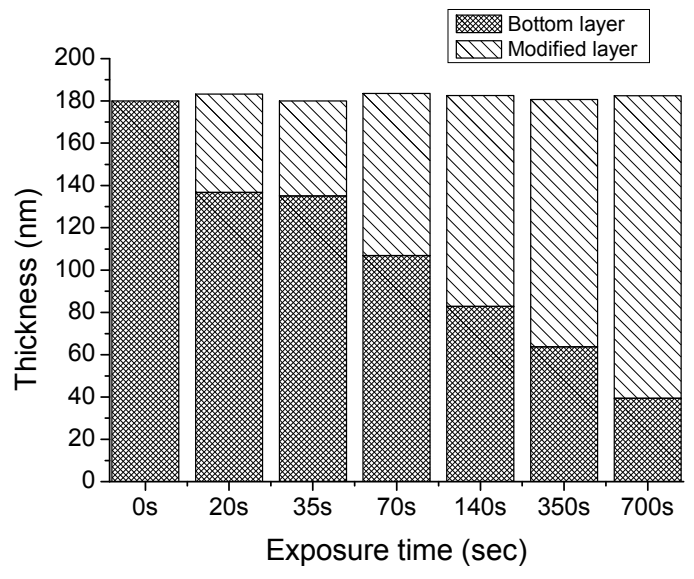

Figure 9. Thickness of the top and bottom layers

The mechanical properties of the modified top layer are depicted in Figure 10. The results show a small reduction of the Young's modulus and hardness of the treated film as the exposure time increases. This change can be attributed to the increase in porosity of the top layer. Nevertheless, the measurements of such a thin film can present a potential effect of the substrate. 


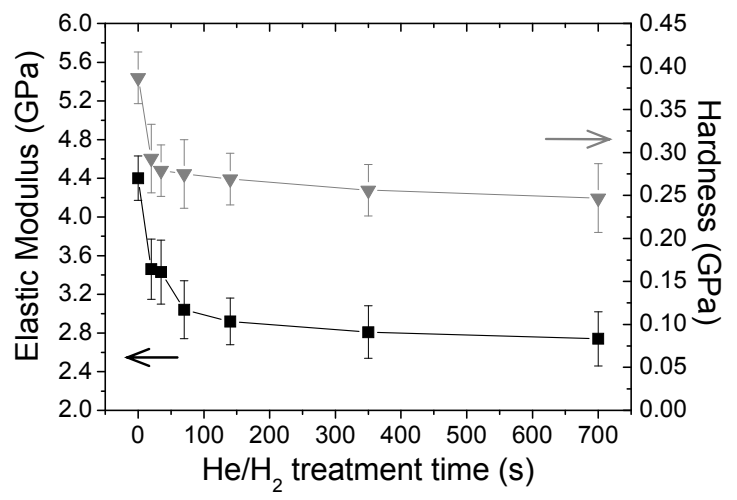

Figure 10. Evolution of $E$ and $H$ with the time exposure as measured by nanoindentation

The variation of the load-displacement curves for different exposure times also explain the decrease of Young's modulus of the top modified layer, considering that the properties of the bottom layer are not modified.

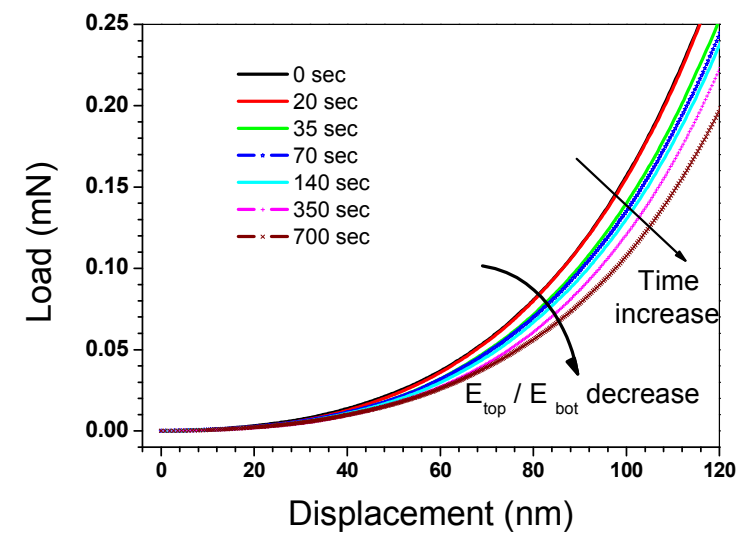

Figure 11. Evolution of the load-displacement curves as function of the exposure time, as obtained by nanoindentation measurements.

In order to understand the influence of the substrate for the different low-k systems, the load-displacement curves of the different systems are simulated. As we do not have the information of the unloading curves, it is not possible to estimate the Young's modulus and yield stress together. For a first estimation we take the Young's modulus measured on the low-k material without the plasma treatment and we varied the yield stress the film. After few modeling iterations, we obtain a good agreement with the experimental result for an yield stress of $96 \mathrm{MPa}$, corresponding to a value of H/4. Figure 12 depicts the measured and the simulated loading curves. The effect of the substrate becomes important after $20 \mathrm{~nm}$ indentation depth. After this value, a big discrepancy is observed in the loading curves.

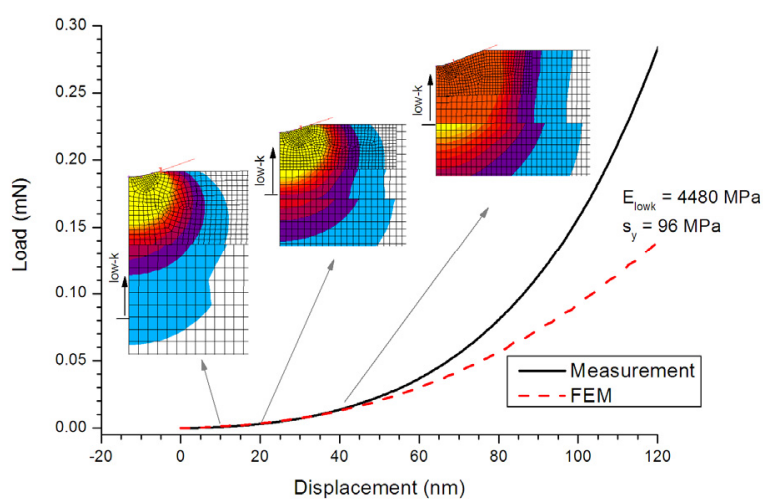

Figure 12. Experimental and FEM load-displacement curves for the reference low-k film $(t=0$ sec). FEM images show the stress distribution in the system during the indentation process.

In the case of double layered system, the mechanical properties of the non-modified low-k film (bottom region) were assumed to be the same as for the single layer. Then the mechanical properties of the modified layer were varied to fit the loading curve. For this case, we have a larger discrepancy between the measured and the calculated mechanical properties. In order to obtain a good fitting with the experimental results, both, the Young's modulus and the yield stress need to be decreased to a value of $1.6 \mathrm{GPa}$ and $54 \mathrm{MPa}$ respectively. The yield stress corresponds to a value of $\mathrm{H} / 5$ (Hardness obtained from the experiments).

Several factors can explain the difference between the measurements and the FEM. (a) As the modified layer is a porous layer, the density of this one change at each penetration depth (closing porous), therefore, a complex stress-strain curve needs to be implemented in the FEM. (b) It is possible that properties of the top layer are not homogeneous in all the thickness, but a gradient of Young's modulus is present. (c) The effect of the substrate may affect the nanoindentation measurements. Figure 13 depicts the measured and the simulated loading curves for the bi-layer system. From the FEM images, it is observed an interaction in the interface between the modified low-k and the nonmodified and also between the low- $\mathrm{k}$ and silicon substrate. This interaction is noticed at indentation depths as low as $10 \mathrm{~nm}$. 


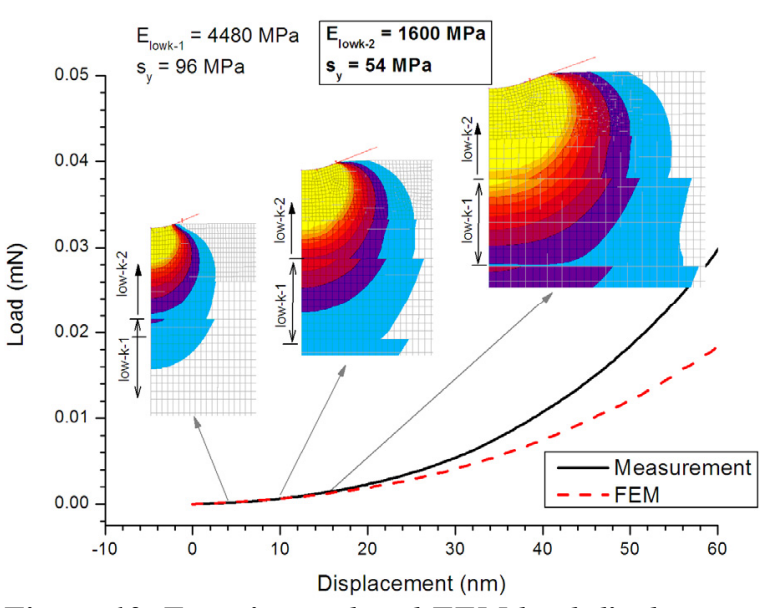

Figure 13. Experimental and FEM load-displacement curves for a low-k film treated during 140 sec. FEM images show the stress distribution in the system during the indentation process.

\section{Conclusions}

This paper explain different issues involved in the analysis of nanoindentation test data such as the influence of the substrate on the nanoindentation measurements, the effects of the elastic modulus and yield stress on the load-displacement curve and the effect of the thin film thickness. It is also shown that FEM can be useful to simulate the nanoindentation test and calculate accurately the mechanical properties of the indented sample by fitting the whole loadindentation depth curve.

The nanoindentation of a double layer low-k dielectric deposited on a silicon substrate was analyzed. The double layer was created by exposing the film to a $\mathrm{He} / \mathrm{H}_{2}$ downstream-plasma, resulting in a modified low-k material in the top region of the film and the original low-k properties in the bottom region.

The measurement of the mechanical properties of the composite film indicates a reduction of the elastic modulus and the hardness as the exposure time increase. However, the measured values of the modified film are overestimated when compared to the FEM. Further parametric studies are necessary to create an accurate stress-strain curve of the modified low-k material.

\section{References}

1. K. Yamanaka, et al, "Low temperature acoustic microscopy for material characterization". Proc. of the IEEE Ultrasonics Symposium. Dec. 1990, Vol.2, pp. 913-920.

2. D. Fabregue, et al. "Multipurpose nanomechanical testing machines revealing the size-dependent strength and high ductility of pure aluminium submicron films". Micro \& Nano Letters, IET. Vol. 2, Issue 1, March 2007, pp. 13-16.
3. Zhi-Guo Li, et al. "The investigation of SAWs propagation in layered anisotropic media for mechanical properties measurement of porous low-k dielectrics". Proc. 8th Solid-State and Integrated Circuit Technology (ICSICT '06), Oct. 2006.

4. Yan-Ting Chen, et al. "Mechanical property measurement of nano-scale metal films on the novel paddle cantilever beams using four step phase-shifting method". Proc. Electronic Materials and Packaging (EMAP 2008). Oct. 2008, pp. 179-182.

5. Oliver, W.C. and Pharr, G.M., "An improved technique for determining hardness and elastic modulus using load and displacement sensing indentation experiments", J. Mater. Res., Vol. 7, no. 3, (1992), pp. 613-617.

6. A. Gouldstone, et al. "Indentation across size scales and disciplines: Recent developments in experimentation and modeling". Acta Materialia Vol. 55 (2007), pp. 4015-4039.

7. F. Carrillo, et al, "Nanoindentation of polydimethylsiloxane elastomers: Effect of crosslinking, work of adhesion, and fluid environment on elastic modulus". J. Mater. Res., Vol. 20, No. 10, Oct 2005. pp. 2820-2830.

8. Rami Haj-Ali et al, "Nonlinear constitutive models from nanoindentation tests using artificial neural networks", International Journal of Plasticity. Vol. 24 (2008), pp. 371-396.

9. A.C. Fischer-Cripps, "Critical review of analysis and interpretation of nanoindentation test data". Surface \& Coatings Technology No. 200 (2006) pp. 4153-4165.

10. J. A. Knapp et al, "Finite-element modeling of nanoindentation", Journal of Applied Physics, Vol. 85, No. 3 (1999), pp. 1460-1474.

11. A.M. Urbanowicz, et al. "Improved Low-k Dielectric Properties Using $\mathrm{He} / \mathrm{H} 2$ Plasma for Resist Removal". Proc. of the Advanced Metallization Conference (AMC), Sept. 2008. 